\title{
Una mirada del proceso de regulación contable internacional en el contexto de globalización
}

\author{
Viviana Pastora Panchi Mayo \\ Universidad Técnica de Cotopaxi, Latacunga, Ecuador \\ Universidad de Los Andes, Programa de Doctorado en Ciencias Contables, \\ Mérida, Venezuela \\ Correo electrónico del autor: vivipanmayo@gmail.com \\ Recepción: 15 Octubre 2017 \\ Aprobación: 11 Diciembre 2017
}

\begin{abstract}
Resumen
La globalización es un proceso que ha inducido transformaciones relevantes en el funcionamiento del sistema económico a nivel mundial. Sus efectos, se precisan en las diversas dimensiones del desarrollo, incluyendo también los aspectos relacionados con el campo contable y financiero. Como un mecanismo para favorecer la competitividad empresarial y, la revelación de información completa y de calidad para la toma de decisiones, el International Accounting Standards Board (IASB) procedió a la formulación de las Normas Internacionales de Información Financiera (NIIF). En función de lo expuesto, este artículo tiene como objetivo disertar en torno a las bases de proceso de regulación contable internacional sustentado por el IASB en el contexto de lo global. Metodológicamente, el trabajo se desarrolló sobre la base del análisis hermenéutico de estudios desarrollados en las líneas de investigación en globalización y regulación contable internacional; así como de la revisión de las NIIF. Las conclusiones de la investigación, revelan la incidencia de lo global en el proceso de regulación contable fundamentado por el IASB, a partir del cual se ha desarrollado la armonización o convergencia contable que promueve la adopción de un mismo lenguaje como base del ejercicio de la contabilidad a nivel mundial.
\end{abstract}

Palabras clave: globalización, contabilidad, regulación contable, NIIF.

REVISTA DE INVESTIGACIÓN SIGMA / Vol. 04, No 1, 2017 (pág. 115-130) 


\title{
A look at the process of international accounting regulation in the globalization context
}

\begin{abstract}
Globalization is a process that has led to significant changes in the functioning of the economic system worldwide. Its effects are specified in the various dimensions of development, including aspects related to the accounting and financial field. As a mechanism to promote business competitiveness and the disclosure of complete and quality information for decision-making, the International Accounting Standards Board (IASB) proceeded to the formulation of the International Financial Reporting Standards (IFRS). Based on the above, this article aims to discuss the basis of the process of international accounting regulation supported by the IASB in the context of the global. Methodologically, the work was developed based on the hermeneutical analysis of studies developed in the lines of research in globalization and international accounting regulation, as well as the revision of IFRS. The conclusions of the investigation reveal the incidence of the global in the process of accounting regulation based on the IASB, from which the harmonization or accounting convergence has been developed that promotes the adoption of the same language as a basis for the exercise of accounting Worldwide.
\end{abstract}

Keywords: globalization, accounting, accounting regulation, IFRS.

\section{Introducción}

A partir de la globalización se han gestado cambios trascendentes en el funcionamiento de los diversos países. Sus efectos trascienden los aspectos económicos para abarcar las dimensiones sociales, políticas, contables y financieras que también forman parte del desarrollo económico y social. Concretamente, en el campo económico y financiero se evidencian como efectos de lo global, lo relacionado con la apertura económica, la ampliación de los mercados, la movilidad de los capitales a nivel internacional, la internacionalización del mercado financiero, entre otros aspectos.

Tal situación ha incidido directamente en la competitividad de las empresas, las inversiones realizadas $\mathrm{y}$, los mecanismos empleados para el desarrollo de las transacciones económicas a nivel mundial. Concretamente en el plano contable y financiero, se procedió a la conformación del International Accounting Standards Board (IASB) como organismo competente para regular la práctica contable internacional en el contexto global, el cual tiene entre sus funciones la emisión de las Normas Internacionales de Información Financiera (NIIF).

Considerando los argumentos expuestos, en este estudio se diserta sobre el proceso de regulación contableinternacional sustentadoporel IASB en el contexto global. Metodológicamente, la investigación se fundamentó en la revisión 
de literatura especializada, empleándose la hermenéutica como técnica orientada hacia el análisis de los trabajos seleccionados en las líneas de investigación en globalización, así como del proceso de regulación contable internacional implementado por el IASB en el contexto global. También, se procedió al análisis de las NIIF emitidas por el IASB.

En estos términos, el trabajo se estructuró en tres secciones. En la primera, se hace referencia al escenario global y su incidencia en el campo contable; en la segunda, se analiza el proceso de regulación contable internacional promovido por el IASB en el contexto global y; finalmente en la tercera, se exponen las conclusiones de la investigación, en las cuales se destaca que aun cuando el proceso de convergencia o armonización contable ha fundamentado la adecuación de las prácticas de la contabilidad a los requerimientos globales, se requiere que el IASB formule y adopte nuevos criterios para estandarizar las definiciones, métodos y criterios empleados para revelar la información de las organizaciones en los mercados altamente globalizados, los cuales exigen para el desarrollo de las transacciones de información de más calidad, útil, confiable y oportuna que favorezca la toma de decisiones.

\section{El contexto global y su incidencia en el campo contable}

En el campo académico existen posturas diversas en torno a la globalización, las cuales se han fundamentado en los aportes de diversas disciplinas como la economía, la sociología, las ciencias humanas, entre otras. El diccionario de la Real Academia Española (2017), la define como un proceso que ha inducido transformaciones profundas en el funcionamiento del sistema económico, incluyendo la ampliación de los mercados. Estos últimos, han adquirido una dimensión mundial que trasciende las fronteras nacionales, pues su dinámica depende cada vez más de los mercados externos y en menor proporción, de las acciones reguladoras de los gobiernos nacionales.

A juicio de Flores (2016) inicialmente la globalización se enfocó como un proceso que permitió la expansión económica de las empresas trasnacionales a nivel mundial. Siguientemente, sus bases se ampliaron para abarcar dimensiones diversas de carácter económico, social, político y tecnológico, entendiéndola como un proceso que integra el conocimiento gestado a nivel mundial $\mathrm{y}$, tiene como referente histórico los cambios generados en los mecanismos empleados para 
abordar procesos y procesar información, de vida de la población. Por ende, la comunidad reconociéndose el factor tecnológico como la internacional debe orientar acciones dirigidas variable clave que fundamenta lo global.

Sobre este aspecto, el Fondo Monetario Internacional (FMI, 2000) establece que la globalización hace alusión a "la creciente integración de las economías de todo el mundo, especialmente a través del comercio y los flujos financieros" (s/p). Esto ha implicado, el libre desplazamiento de personas (mano de obra), la transferencia de conocimientos (tecnología), factores productivos, así como el intercambio de bienes y servicios en los mercados internacionales. En función de lo expuesto, la globalización ha supuesto un fortalecimiento de la integración de los países para participar en el comercio internacional, incluyendo también el caso de los mercados financieros.

En el documento citado, se enfatiza que el proceso global ha contribuido con el mejoramiento del bienestar social en la mayoría de los países, destacando que los más beneficiados han sido los países desarrollados. Así mismo, se precisa que los países en desarrollo, han encontrado limitaciones para integrarse al contexto global como consecuencia de las estrategias empleadas para alcanzar el objetivo de mejorar la calidad 
el resto de países que conforman el contexto de la "globalidad".

Con relación a este aspecto, Castells (2002) explica que la perspectiva económica de la globalización, no implica la integración total de las economías, sino más bien que sus actividades se articulan a una especie de núcleo globalizado, cuyo funcionamiento se concibe como una unidad a nivel mundial. Dentro de estas actividades, el autor destaca el caso de los mercados financieros, los cuales aun cuando en algunos casos no presentan un carácter globalizado, siempre se articulan al mercado financiero global.

Así mismo, también se encuentran globalizados los procesos científicos $\mathrm{y}$ tecnológicos que fundamentan la innovación de tecnología en cuanto a procesos, productos y servicios, la fuerza de trabajo especializada, el funcionamiento de las corporaciones multinacionales y, las redes de empresas asociadas con éstas. Por tanto, económicamente lo que caracteriza la globalización es precisamente que “el comercio internacional está cada vez más interrelacionado funcionando en un mercado global" (Castells, 2002, p. 46).

Como ejemplo de las transformaciones económicas ocurridas en el contexto global,
Castell (2002) cita el caso de los mercados financieros, los cuales se han integrado a través de los siguientes aspectos: i) los precios de los productos financieros se cotizan a nivel internacional en función del comportamiento del mismo en un país determinado; ii) las plataformas tecnológicas y las tecnologías de información y comunicación, permiten la transferencia de grandes capitales a nivel mundial de manera casi instantánea, lo cual no resulta un proceso controlado por parte de los países donde se localizan las empresas que participan en las transacciones financieras a nivel internacional y; iii) la certificación financiera, entendida como un proceso que permite a las empresas evaluadoras de países, organizaciones y mercados, subir o bajar sus posiciones de acuerdo con el funcionamiento del mercado global.

En el campo social, la globalización ha implicado un cambio en las relaciones sociales, pues el desarrollo de las tecnologías de información y comunicación han fundamentado la "inter culturalización" sobre la base de los productos, servicios y relaciones establecidas entre un país y el resto del mundo. En tal sentido, la participación de diversos países en la producción y distribución de un mismo bien o servicio, ha inducido una división mundial del 
trabajo, así como la especialización de factores de producción en zonas que se encuentran geográficamente distanciadas, lo cual ha permitido gestar "relaciones internacionales de producción y 'movilidad de factores productivos' que da cuenta de las movilizaciones humanas en busca de nuevas formas de desarrollo profesional o económico" (Flores, 2016, p. 40).

En el plano político, la globalización ha fundamentado la conformación de una nueva sociedad, cuyas bases han conducido hacia el establecimiento de nuevas relaciones políticas, sociales y económicas entre los Estados. En esta dirección Flores (2016) enfatiza en la concepción de "estado trasnacional", la cual resulta adecuada para definir un Estado que se encuentra altamente influenciado en el contexto global por la dinámica y funcionamiento de otros Estados.

En el marco de estas transformaciones, cabe destacar que lo contable se ha vinculado expresamente con el aspecto económico y financiero. Como lo expresa Rueda (2010) se ha enfatizado en la necesidad de adoptar estándares internacionales de contabilidad que además de favorecer la libre circulación del capital en los mercados financieros mundiales, orienten la inserción competitiva de las economías nacionales en la nueva lógica mundial del capitalismo financiero.

Tal postura ha sido ampliamente fundamentada por el International Accounting Standards Board (IASB) que en el cumplimiento de las funciones reguladoras de la práctica contable a nivel internacional, promueve el paso de una contabilidad de alcance nacional a una de carácter global. Es decir, de una contabilidad que además de favorecer la competitividad de las empresas en los mercados internacionales, satisfaga la necesidad de información financiera de los distintos usuarios que participan en las transacciones de las distintas empresas localizadas a nivel mundial (Rueda, 2010).

De este modo Rodríguez, De Freitas y Zaa (2012) manifiestan que producto de la globalización, la práctica contable ha evolucionado pues ésta no se limita simplemente a la revelación de información financiera que no reporta valor agregado para las empresas y organizaciones, sino que se orienta más bien hacia las consultorías y asesorías gerenciales, que aporten a los usuarios internos y externos de la información, soluciones completas y efectivas a los problemas que limitan el desarrollo organizacional. 
Por ende, como lo afirma Talla (2012) en el contexto global, la contabilidad además de fundamentar la revelación de información completa para la toma de decisiones, debe permitir el acceso a la información financiera internacional, al conocimiento sobre el funcionamiento de otros mercados, así como de las bases que sustentan el desarrollo industrial. Tales objetivos podrán cubrirse, en la medida que la contabilidad aporte información financiera que revele la situación de la empresa bajo un mismo lenguaje, empleando metodologías uniformes que sustenten la comparación de la misma por parte de los diversos países y organismos.

En síntesis, puede afirmarse que como consecuencia de la globalización se han inducido transformaciones profundas en el plano contable. Como lo afirma Jaramillo \& Cárdenas-Pérez (2018), el sistema financiero uno de los factores más importantes dentro de la economía, puesto que el mismo permite canalizar el ahorro hacia la inversión, siendo el pilar fundamental para la generación de riqueza, por tanto esto debe ir de la mano del proceso de armonización fundamentado por el IASB a través de la emisión de las Normas Internacionales de Información financiera (NIIF), las cuales constituyen los postulados normativos que rigen el funcionamiento de la práctica contable a nivel internacional.

\section{Las bases de la regulación contable fundamentada por el IASB en el contexto global}

En el contexto global, la información financiera revelada por las empresas debe reunir un conjunto de atributos relacionados principalmente con la objetividad, la homogeneidad y la comparabilidad de la misma. Estos fundamentan la presentación de una información financiera útil para la toma de decisiones. De este modo, a nivel mundial el IASB, en cumplimento de su función reguladora del ejercicio contable ha comenzado a promover el proceso de regulación contable a nivel internacional, entendido en los términos de Vargas, Diossa y Lotero (2014) como un proceso social orientado a determinar la sujeción de algún o algunos aspectos a un conjunto de reglas formuladas para normalizar su comportamiento.

Sobre este aspecto, los autores citados exponen que el realismo constituye la base del modelo de regulación adoptado en contabilidad. A partir de sus fundamentos, se han estructurado las metodologías empleadas en el ejercicio práctico para la cuantificación, reconocimiento y medición de los distintos fenómenos que conforman el hecho contable como objeto de 
estudio de esta disciplina.

Otra postura al respecto, la presentan Molina y Tua (2010) al enfatizar que el proceso de regulación además de abarcar la emisión de la normatividad que favorece el empleo de estándares contables para la caracterización, reconocimiento y valoración de las transacciones financieras, contempla también los aspectos que fundamentan la emisión de juicios profesionales durante el proceso de auditoría.

En esta dirección cabe acotar que históricamente, el proceso de regulación contable ha evolucionado para fundamentar la emisión de las normas que regulan la práctica de manera más ajustada para revelar la realidad financiera de las organizaciones. Como lo afirma Tua (1995), tal proceso "no ha utilizado siempre los mismos criterios, sino que, a lo largo de su devenir histórico, ha manejado diferentes concepciones, en un continuo esfuerzo por encontrar el itinerario lógico más adecuado para la construcción de reglas contables" (p.17). Así mismo, el autor precisa que Estados Unidos ha sido uno de los países que ha fundamentado la normalización contable a nivel internacional, pues los estándares estadounidenses han ejercido una influencia determinante sobre el proceso de regulación contable adoptado para el resto del mundo.

Sobre este aspecto, Zeff (1999) apunta que los antecedentes de un "Marco Conceptual" en la literatura contable norteamericana se atribuyen a los esfuerzos realizados por Patón y Canning. Concretamente en la propuesta de Patón denominada Accounting Theory, el autor presenta una reformulación de la teoría contable, ajustándola a las condiciones y necesidades de la empresa mercantil también conocida como la gran empresa. Fundamentándose en el argumento expuesto, Patón propone un conjunto de postulados que aportaron las bases para estructurar los fundamentos de la Contabilidad moderna.

Retomando el planteamiento de Tua (1995), en el proceso de regulación contable estadounidense se precisan claramente tres etapas. La primera, se extiende hasta 1959, con la creación del Accounting Principles Board (ABP) y, se orientó hacia la búsqueda y aceptación generalizada de las manifestaciones del proceso de regulación norteamericano; la segunda de carácter lógico, abarcó el período comprendido entre 1959 y 1973 cuando se procede a la constitución del Financial Accounting Standards 
Board (FASB) y; la tercera de orientación teleológica, precisada entre 1973 hasta el momento que se ha encauzado básicamente hacia la discusión de los objetivos relacionados con la información financiera, incluyendo lo relacionado con el paradigma de la utilidad.

En este sentido, Tua (1995) expresa que el proceso de regulación se ha caracterizado en los siguientes términos: a) en la etapa de aceptación generalizada, se encaminó hacia la homogenización de las prácticas contables, empleándose el término "principio contable" como un sinónimo de norma de acepción general o "norma comúnmente aceptada"; b) en la etapa lógica, se procedió a la introducción del método deductivo como base para la generación de las construcciones contables (reglas) que regularían el ejercicio de la contabilidad, afianzándose el término "principio contable", como una especie de "macro regla" que orientaría la práctica de la contabilidad y; c) en la etapa teleológica, se enfatiza en los requerimientos de la información financiera en función de las necesidades de los usuarios, formulándose un conjunto de características cualitativas de la información que resultan fundamentales para el cumplimiento de los objetivos de la contabilidad.
Como bien se afirmó en los párrafos precedentes, durante la tercera etapa se gestan las bases del proceso de regulación actual. Concretamente, en 1973 se procedió a la creación del IASC (International Accounting Standards Committee/ Comité de Normas Internacionales de Contabilidad) bajo un acuerdo de los profesionales contables que representaron los siguientes países: Alemania, Australia, Estados Unidos, Francia, Holanda, Irlanda, Japón, México y el Reino Unido. Este organismo, se creó con competencias para formular normas contables de amplia aceptación y aplicación en los distintos países, favoreciendo la armonización de la información financiera y su comparabilidad a nivel internacional (IFRS, 2018).

Posteriormente en el año 2000, como un mecanismo para promover la convergencia entre las normas nacionales e internacionales de contabilidad, se procedió a la creación del InternationalAccounting Standards Board(IASB) conocido como Junta de Normas Internacionales de Contabilidad en sustitución del Comité de Normas Internacionales de Contabilidad (IASC). Tal proceso de convergencia, lo ha fundamentado el IASB partir de la formulación de las Normas Internacionales de Información financiera (NIIF), las cuales promueven el uso de un mismo 
lenguaje contable a nivel mundial, de manera que se puedan adoptar los mismos criterios y métodos para revelar la información financiera por parte de las organizaciones que participan en los mercados globales.

En tal sentido, en el Prólogo de las NIIF (IASB, 2012) se establecen como objetivos del IASB, el cumplimiento de las siguientes funciones:

(a) Formular un conjunto de Normas de Información Financiera ampliamente aceptadas, las cuales deben fomentar la presentación de información comparable, transparente y de alta calidad, como aspectos que favorecen la toma de decisiones por parte de los inversionistas y demás actores que participan en los mercados de capitales.

(b) Promover el uso y aplicación de las NIIF como sustento del proceso de regulación contable a nivel mundial.

(c) Favorecer la adopción de las NIIF a través de un proceso de convergencia entre las normas de contabilidad nacionales y las NIIF.

En tal dirección, el principal objetivo del proceso de regulación contable internacional orientado por el IASB, ha consistido en promover la adopción de un mismo lenguaje contable a nivel mundial. Esto se materializa en la medida que se procede a la estandarización de los procedimientos adoptados como fundamento de la práctica contable. Es decir, de los “criterios, reconocimiento y valoración de los elementos financieros correspondientes al desarrollo empresarial" (Vargas, Diossa y Lotero, 2014, p. 9).

Puede afirmarse entonces, que la regulación contable internacional ha favorecido la unificación de los conceptos, métodos y prácticas que conforman el lenguaje contable, para formular un marco regulatorio que resulte aplicable para todos los países que acepten el proceso de convergencia para la adopción de las NIIF. Como lo expresan Vargas, Diossa y Lotero (2014) esto ha implicado para las diversas naciones, transformar las normas de contabilidad locales en función de los requerimientos de los estándares internacionales propuestos en las NIIF.

En esta orientación, Sunder (1997) apunta que la normatividad a nivel contable no se diferencia de otras categorías de normas que regulan el funcionamiento de la sociedad. Ambas orientan "el comportamiento individual hacia 
alguna situación considerada deseable por algún criterio de elección social, o sirven de guía para la coordinación entre los individuos” (p. 259).

Desde esta postura, el proceso de convergencia contable puede entenderse como una actividad regulada y organizada, que mediante la emisión de normas favorece la consecución de comportamientos homogéneos en la revelación de la información financiera. Esta última se concibe, bajo el Marco Conceptual de las NIIF (IFRS, 2010) como información útil a los “inversores, prestamistas y otros acreedores existentes y potenciales para tomar decisiones sobre el suministro de recursos a la entidad" (p.OB2).

Es decir, la utilidad de la información financiera se expresa en los términos de la calidad de la misma para promover la toma de decisiones en un contexto global que resulta socialmente compartido y ampliamente competitivo. $\mathrm{Al}$ respecto De Larrea (1997), expresa que tal información debe ser objetiva, veraz, útil y confiable, lo cual permite la "comparabilidad de la información con diferentes puntos de referencia para que sea aceptada y utilizada equitativamente para la toma de decisiones" ( $p$. $50)$.
Además de los aspectos descritos, De Larrea (1997) apunta que la información financiera debe concebirse como una información de calidad. Es decir "la que demandan los que requieren del uso de ella como herramienta elemental para tomar decisiones en un contexto ampliamente compartido y muy competido" (p. 50). De este modo, en función de la relación establecida entre la información financiera y los usuarios de la misma, la primera adquiere un valor estratégico para promover la inserción competitiva de las organizaciones en los mercados globales.

No obstante, como bien lo afirma De Larrea (1997) para que la información financiera se considere competente, de calidad $\mathrm{y}$, represente un valor fundamental para mejorar la competitividad empresarial, debe tener un valor vigente por su carácter útil y confiable. Para la autora, la primera cualidad mencionada se relaciona con la información; mientras que la segunda, se vincula directamente con el usuario o los usuarios que requieren la información como base de la toma de decisiones.

Basándose en el enfoque de la utilidad de 
la información financiera, el Marco Conceptual de las NIIF (IFRS, 2010) destaca la relevancia y la representación fiel, como cualidades clave para la toma de decisiones. Así mismo, se precisa que la información resulta útil en la medida que pueda ser comparable, verificable, oportuna y comprensible. En función de las cualidades de la información financiera como base de la estandarización del leguaje contable, el IFRS (2010) procedió a la distinción entre características cualitativas fundamentales y características cualitativas de mejora.

Las fundamentales, se expresan a través de: a) la relevancia que fundamenta la capacidad de la información de influir en la toma de decisiones, lo cual depende de su valor predictivo, confirmatorio o de ambos y; b) la representación fiel, que implica la capacidad de la información financiera para representar "fielmente" los fenómenos u hechos que se pretenden representar. Ambas características, sustentan que la información financiera para ser útil debe caracterizarse al mismo tiempo por la relevancia y la representación fiel, pues "Ni una representación fiel de un fenómeno irrelevante ni una representación no fidedigna de un fenómeno relevante ayuda a los usuarios a tomar decisiones adecuadas" (IFRS, 2010, p. CC17).
Sobre este aspecto, Gómez (2004)

expresa que el IASB como organismo emisor de las NIIF, debe orientar acciones para el alcance de un equilibrio entre las cualidades cualitativas fundamentales, de manera que la revelación de la información represente "en forma fiel la sustancia económica de las transacciones y que, a su vez, es lo suficientemente comprensible para los inversionistas y demás interesados en la información" (p. 59).

Así mismo, las características cualitativas de mejora de la información se fundamentan en: a) la comparabilidad, que promueve la utilidad de la información en la medida que ésta pueda comparase con información similar revelada tanto por otras entidades, como por la misma entidad para otros períodos financieros; b) la verificabilidad, que asegura a los usuarios el acceso a una información que representa fielmente los hechos económicos de la entidad; c) la oportunidad, que implica disponer de información financiera en el momento oportuno para influir en la toma de decisiones y; d) la comprensibilidad, que promueve la revelación de información financiera clara y concisa. Estas características, deben maximizarse para mejorar la utilidad de la información financiera. No obstante, como bien se apunta en el Marco Conceptual de las NIIF 
(IFRS, 2010) "las características cualitativas de mejora, individualmente o en grupo, no pueden hacer la información útil si es irrelevante y no se representa fielmente" (IFRS, 2010, p. CC 33).

Aun cuando las características cualitativas fundamentales y de mejora expuestas, han contribuido con la unificación o estandarización del leguaje contable, se requieren otros criterios y metodologías más adecuadas para abordar la subjetividad que caracteriza el hecho contable en el contexto global. En tal dirección, Pérez (2010) manifiesta que la contabilidad se enfrenta actualmente al reto de "encontrar unos criterios contables que aborden la complejidad con que hoy en día se desarrolla el mundo de los negocios, y además que sean válidos y aceptados universalmente" (p. 45). No obstante, el autor también reafirma que la adopción de nuevos criterios para el reconocimiento y valoración de la información, no aportarán soluciones cuando las deficiencias encontradas son producto del incumplimiento voluntario de la normatividad por parte de los encargados de revelar la información financiera.

Este proceso de convergencia, también implica cambios relacionados con el juicio profesional emitido por los profesionales encargados de preparar y auditar la información financiera, pues en ambos casos éstos requieren "demostrar que sus juicios e interpretaciones fueron realizados de buena fe y apegados a los pronunciamientos vigentes" (Gómez, 2004, p. 59).

Por tanto, a partir de la convergencia para la incorporación de las NIIF como normatividad de base para regular el ejercicio contable, los profesionales en este campo deben capacitarse para captar la esencia del hecho económico que conforma la realidad abordada por la contabilidad en el contexto global. Esto, permitiría analizar e interpretar las distintas dimensiones (objetivas y subjetivas) que hacen parte de la contabilidad, aportando respuestas para la solución de problemas complejos. En palabras de Gómez (2004) "será imperativo que los profesionales de la contabilidad conozcan más acerca de las teorías y técnicas de valuación existentes" ( $\mathrm{p}$. 59).

A manera de síntesis, conviene destacar que el proceso de convergencia contable internacional, ha promovido algunos cambios trascendentales en el proceso de regulación contable a nivel internacional. Entre éstos se encuentran: a) la adopción de una normatividad contable a nivel mundial que resulta ampliamente compatible y socialmente aceptable como base 
para armonizar la normativa nacional a las exigencias internacionales; b) la estandarización de los criterios y métodos empleados para la revelación de la información financiera que muestra la realidad de la situación de una entidad durante un período financiero y; c) facilitar la comparabilidad de la información financiera entre las distintas organizaciones, así como entre los distintos períodos financieros de una misma entidad.

\section{Conclusiones}

La globalización de la economía ha implicado efectos diversos en el desarrollo económico y social. Como muestra de esta situación, se precisan a nivel mundial transformaciones relevantes en las dimensiones económicas, sociales e institucionales asociadas al desarrollo. Esto también se ha reflejado en el campo contable y financiero, pues como fundamento para la inserción competitiva de las empresas en los mercados internacionales, se requiere la revelación de información financiera completa y de calidad, como cualidades clave que favorecen la comparabilidad de la misma al momento de tomar decisiones.

En esta dirección, el IASB como organismo encargado de regular la práctica contable a nivel mundial, ha fundamentado el desarrollo de un proceso de armonización o convergencia contable, para la adecuación de la normatividad nacional a los requerimientos del nuevo marco regulatorio fundamentado en las NIIF, bajo el cual la información financiera resulta útil en la medida que se basa en las características cualitativas fundamentales precisadas en la relevancia y la representación fiel; así como en las características cualitativas de mejora concretadas en la comparabilidad, verificabilidad, oportunidad y comprensibilidad

Las características mencionadas, han fundamentado la adopción de un lenguaje contable estandarizado por parte de los países que han entrado dentro del proceso de armonización. Esto ha implicado en el ejercicio de la práctica profesional, la unificación de las definiciones, métodos y criterios empleados para revelar la realidad contable de las organizaciones.

No obstante, aun cuando se han presentado avances en el campo contable para incorporar las exigencias de lo global en los requerimientos de la información financiera, también se requiere que el organismo regulador formule nuevos criterios para sustentar el abordaje del hecho económico objeto de estudio de la contabilidad desde una postura más integradora, pues la realidad 
contable no puede continuar analizándose desde una visión netamente objetiva, sin considerar los aspectos subjetivos que también forman parte de la misma, los cuales resultan fundamentales para revelar la verdadera situación de las empresas en el contexto global.

\section{Referencias bibliográficas}

Castells, M. (2002). La era de la información: economía, sociedad y cultura. La Sociedad red. Madrid: Alianza Editorial. Disponible: http://www.felsemiotica.org/ site/wp-content/uploads/2014/10/LA_ SOCIEDAD_RED-Castells-copia.pdf

Castells, M. (2000). Globalización, sociedad y política en la era de la información. Bitácora [Revista en línea],(4)1, 42-53. Disponible: file://C:/Documents\%20and\%20Settings/ usuario/Mis\%20documentos/Downloads/ Dialnet-GlobalizacionSociedadYPolitic aEnLaEraDeLaInformaci-4008342.pdf [Consulta: 2017, noviembre 23]

Flores, M. (2016). La globalización como fenómeno político, económico y social. Orbis [Revista en línea], 12(34), 2641. Disponible: http://www.redalyc.org/ pdf/709/70946593002.pdf[Consulta: 2017, noviembre 23]

Fondo Monetario Internacional. (2000). La globalización: ¿Amenaza u oportunidad? [Documento en línea]. Disponible: https://www.imf.org/external/np/exr/ ib/2000/esl/041200s.htm[Consulta: 2017, diciembre 05]

Gómez, D. (2004). Reglas Vs. Principios. Ejecutivos de finanzas [Revista en línea], 56-59. Disponible: file:///F:/Conta/ Reglas\%20vs\%20principios.pdf[Consulta: 2018, enero 6]

IFRS. (2018). NIC NIFF. Portal en español de
Normas Internacionales de Información Financiera NIIF - IFRS. Disponible: http:// www.nicniif.org/home/iasb/que-es-el-iasb. html[Consulta: 2018, diciembre 26]

IFRS. (2012). Prólogo de las Normas Internacionales de Información Financiera. IFRS Foundation. Disponible: http://www.confecooporiente.com/web/ images/pdf/Prologo \%20a\%201as\%20 NIIF\%20_part\%20a_165.pdf[Consulta: 2018, enero 5]

IFRS. (2010) El Marco conceptual para la información financiera. Disponible en: http://www.leyes.com.py/ documentaciones/infor_interes/ contabilidad/NIIF/marco_conceptual_niff. pdf[Consulta: 2018, enero 5]

Jaramillo, M.; Cardenas, A. (2018). Modelización econométrica bajo la metodología de BoxJenkins. Estudio empírico a la liquidez del sistema financiero ecuatoriano. Investigación Operacional, 39(4), 592607.

Molina, H.; Tua, J. (2010). Reglas versus Principios contables, ¿son modelos incompatibles? Revista Española de Financiación y Contabilidad [Revista en línea], (XXXIX)146, 259-287. Disponible: http://aeca.es/old/refc_19722013/2010/146-2.pdf[Consulta: 2018, enero 3]

Larrea, E. (1997). La homologación internacional de la normatividad contable. Revista de Contaduría y administración, (184), 4766. Disponible: file:///F:/Conta/La $\% 20$ homologación $\% 20$ internacional $\% 20$ de $\% 201 a \% 20$ normatividad $\% 20$ contable. pdf[Consulta: 2018, enero 3]

Pérez, J. (2010). Una verdadera evolución; efectos contables de a convergencia financiera mundial. CISS Técnica contable [Revista en línea], (731), 44-52. Disponible: file://F:/Conta/tecnicacontable_jorge_ 
perez3.pdf[Consulta: 2018, enero 5]

Real Academia Española. (2016). Diccionario de la lengua Española. [Documento en línea]. Disponible: http://dle.rae.es/[Consulta: 2017, diciembre 22]

Rodríguez, J.; De Freitas, S.; Zaa, J. (2012). La contabilidad en el contexto de la globalización y la revolución teleinformática. Análisis de coyuntura [Revista en línea], (XVIII) 1, 161-183. Disponible: file://C:/Users/PS-5100/ Downloads/3347-7557-1-SM\%20(2).pdf

Rueda, G. (2010). El papel de la contabilidad ante la actual realidad económica, social y política del país. Más allá de la convergencia de prácticas mundiales. Cuadernos de Contabilidad [Revista en línea], (11)28, 149-169. Disponible: file:///C:/Users/usuario/Documents/ v11n28a06.pdf[Consulta: 2017, noviembre 25]

Sunder, S (1997). Teoría de la Contabilidad y el Control. Colombia: Universidad Nacional de Colombia.

Talla, S. (2012). La globalización y la armonización contable. Quipukamayoc [Revista en línea], 20(35). Disponible: http://sisbib.unmsm.edu.pe/bibvirtual/ publicaciones/quipukamayoc/1999/ primer/global.htm[Consulta: 2017, diciembre 26]

Tua, J. (1995). Lecturas de teoría e investigación contable. Colombia: Centro interamericano Jurídico- Financiero.

Vargas, Diossa y Lotero (2014). Una aproximación a la tensión entre realismo y regulación contable internacional. XIX Congreso Internacional de Contaduría, Administración e Informática. México. Disponible: http://premio.investiga.fca. unam.mx/docs/ponencias/2014/3.1.pdf[Co nsulta: 2017, diciembre 26]
Zeff, S. (1999). La Evolución del marco conceptual para las empresas mercantiles en Estados Unidos. Revista española de financiación y contabilidad [Revista en línea], (100), 151-194. Disponible: file://C:/Users/PS-5100/Downloads/ Dialnet-LaEvolucionDelMarcoConcep tualParaLasEmpre sas Mercan-44297. pdf[Consulta: 2017, octubre 25] 